\title{
Double Emergency Pulmonary Embolism and Aortic Dissection: About a Clinical Case
}

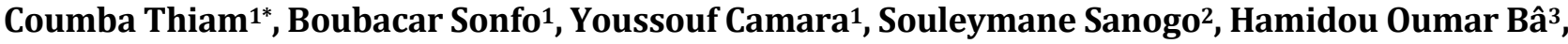 \\ Sidibé Samba4, Massama Konaté5, Asmaou Keita6, Sako Mariam4, Mahamadou Cissé1, \\ Ibrahima Sangaré ${ }^{3}$, Massama Camara ${ }^{1}$, Menta Ichaka ${ }^{3}$
}

${ }^{1}$ Cardiology Department of CHU Kati, Bamako, Mali

${ }^{2}$ Imaging Department, CHU Mère-Enfant, Bamako, Mali

${ }^{3}$ Cardiology Department, CHU Gabriel Touré, Bamako, Mali

${ }^{4}$ Cardiology Department, CHU Point G, Bamako, Mali

${ }^{5}$ Internal Medicine Service, Mali Hospital, Bamako, Mali

${ }^{6}$ Cardiology Department, CHU Mère-Enfant, Bamako, Mali

Email: ^thiampoupe@yahoo.fr

How to cite this paper: Thiam, C., Sonfo, B., Camara, Y., Sanogo, S., Bâ, H.O., Samba, S., Konaté, M., Keita, A., Mariam, S., Cissé, M., Sangaré, I., Camara, M. and Ichaka, M. (2020) Double Emergency Pulmonary Embolism and Aortic Dissection: About a Clinical Case. World Journal of Cardiovascular Diseases, 10, 550-557. https://doi.org/10.4236/wjcd.2020.108054

Received: June 23, 2020

Accepted: August 17, 2020

Published: August 20, 2020

Copyright $\odot 2020$ by author(s) and Scientific Research Publishing Inc. This work is licensed under the Creative Commons Attribution International License (CC BY 4.0).

http://creativecommons.org/licenses/by/4.0/ (c) (i) Open Access

\begin{abstract}
Introduction: Pulmonary embolism and aortic dissection are two formidable cardiovascular emergencies. Their exceptional association has a poor prognosis with very high mortality. The aim of our study was to report the case of a 31-year-old patient with pulmonary embolism associated with De Bakey's type I aortic dissection, admitted to the cardiology department of Kati University Hospital. Case Presentation: We report the case of a young patient of 31 with no known cardiovascular history, on estrogen-progestogen contraception for 10 years, who consults for a left basal thoracic pain of increasing intensity, a whitish productive cough, hemoptysis and dyspnea stage III. She was admitted to the cardiology department, transthoracic cardiac ultrasound objectified aortic insufficiency, dissection of the aorta, the presence of the intimal flap, the false and the true channel, dilation of the right cavities with HTAP. The chest CT scan revealed bilateral lobar and segmental pulmonary embolism, De Bakey's type I aortic dissection. Medical treatment was instituted for hemodynamic stabilization at the end of sending her to a center specializing in cardiovascular surgery for better management; unfortunately she succumbed before the preparations for her evacuation were finished. Conclusion: The pulmonary embolism associated with aortic dissection constitutes a medical emergence of rare incidence in a cardiological environment; if the diagnosis was quickly made in our patient, the lack of technical platform made management difficult.
\end{abstract}

\section{Keywords}

Pulmonary Embolism, Aortic Dissection, CHU, Kati 


\section{Introduction}

Pulmonary embolism is defined by the occurrence of an acute or subacute, partial or total occlusion of the trunk or of one of the branches of the pulmonary artery by a circulating foreign body; most often a fibrino-cruoric clot migrates from deep vein thrombosis. In recent decades, specific diagnostic tests, including lung scintigraphy and angiography, have helped define a category of less severely affected patients with non-fatal pulmonary embolism. There are thus variable clinical forms of pulmonary embolism depending on the severity, ranging from small thrombosis without clinical translation, to a massive, sudden and fatal form. Pulmonary embolism is still a major challenge in medicine today despite advances in prevention, diagnosis and treatment. It is often underestimated, under diagnosed and therefore under treated [1]. It is always to be considered as a frequent and serious disease [2], especially in hemodynamically unstable patients [3], in the elderly [4] or in patients with severe underlying pathology in particular cardiopulmonary disease [5] [6]. Aortic dissection consists of a rupture of the intima of the aorta, the innermost thin layer of the wall of this vessel, often over half of its circumference. The presence of a degeneration of the middle part, called the media, also appears necessary. This rupture usually occurs in the right lateral wall of the aorta, where the hydraulic shear by the pressure wave is maximum, or just after the arterial ligament in the descending aorta. The pulsatile aortic flow dissects the elastic lamellar layers of the vessel by creating a false light, inside the wall. This spreads distally in most cases, but can also progress proximally towards the heart. It can even recreate one or more intimate breaches to return to the true aortic light. Acute aortic dissection is described as rare. Aortic dissection mainly affects elderly patients with chronic hypertension. Its occurrence before 40 years should lead to research into the intake of toxic drugs, in particular cocaine, as well as the presence of Marfan syndrome. Pulmonary embolism and acute aortic dissection are two formidable cardiovascular emergencies. Their exceptional combination has a poor prognosis with very high mortality in the absence of prompt surgical management. The association of the two pathologies, the rarity in a young subject, motivated us to report this case.

\section{Observation}

This is a 31-year-old female patient with no known cardiovascular history, having been on estrogen progestogen contraception for 10 years, family history of hypertension and diabetes. She was seen in a cardiology consultation for increasing basal left thoracic pain without lulling factors or triggers, a whitish productive cough, hemoptysis and dyspnea stage III. The physical examination had found a weight $=68.5 \mathrm{~kg}$, the height $=1.85 \mathrm{~m}$, a turgor of the jugular veins, the general condition was fair, the extremities are fine, broad and deformed, the thorax deformed, the flat feet (marfanoid appearance), hyperpulsatility of the arteries of the neck, tip shock visible in the $5^{\text {th }}$ intercostal space left, a systolic breath grade IV/VI in the mitral focus with a tremor, a systolic breath grade II in 
the aortic focus, a burst of the second noise at the pulmonary focus, a tachycardia at $120 \mathrm{BPM}$, a blood pressure at $110 / 60 \mathrm{mmHg}$, a respiratory frequency at 40 cycles $/ \mathrm{min}, \mathrm{SO}_{2}=80 \%$, a reduction in vesicular murmurs at the right pulmonary base, presence of crackling groans at the left pulmonary base. On the basis of these clinical signs an admission has been proposed Chest radiography revealed an enlargement of the cardio-mediastinal silhouette with the bulging left middle arch (Figure 1). The electrocardiogram revealed a sinus tachycardia, the transthoracic cardiac ultrasound found dilation of the ascending aorta (Figure 2(A)), the intimal flap (Figure 2(B)), the intimal flap (Figure 3(A)), the intimal flap at the aorta descending (Figure 3(B)). The thoracic CT scan showing a dissection of the ascending aorta (Figure 4-red arrows) and the descending thoracic aorta (Figure 4-yellow arrows), a pleural effusion of low abundance (Figure 4(e)), thrombi in the bilateral lower lobar arteries (Figure 5-yellow arrows) and pleural effusion (Figure $5(\mathrm{e})$ ). The hemogram showed anemia $(\mathrm{Hb}=10.9 \mathrm{~g} / \mathrm{dl}$; Hcte $=32.4 \%$; $\mathrm{MCV}=72.3 \mathrm{fl} ; \mathrm{MCH}=24.2 \mathrm{Pg} ; \mathrm{MCHC}=33.5 \mathrm{~g} / \mathrm{dl})$, leukocyte $=6.4810^{3} / \mathrm{mm}^{3}$; monocyte $=336 \%$; basophil $=374 \%$. The biochemical assessment presented a hyper cretininemia (168.17 umol/L), transaminases and the ionogram were normal.

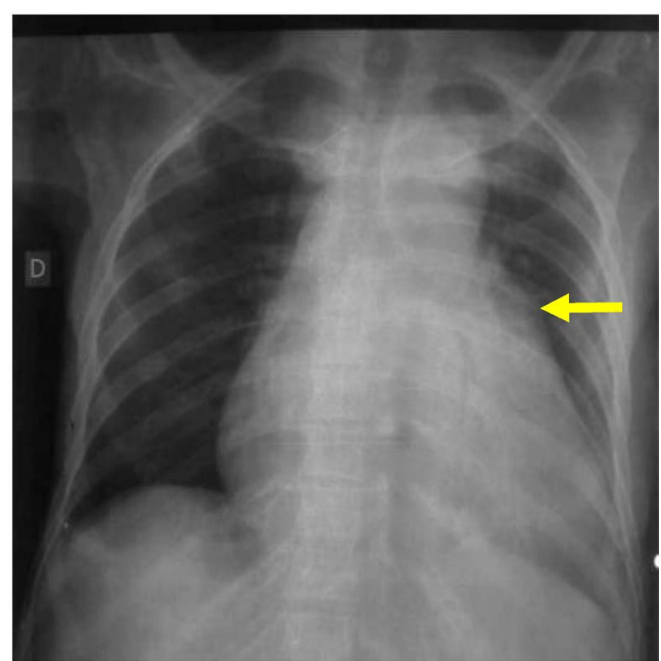

Figure 1. Frontal chest X-ray showing a widening of the cardio-mediastinal silhouette with the bulging left middle arch (yellow arrows).
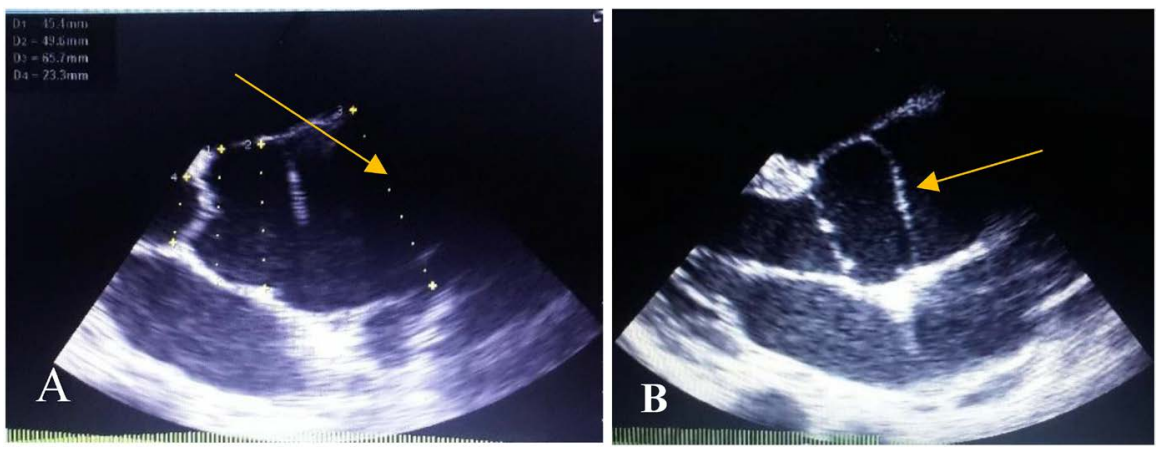

Figure 2. Transthoracic ultrasound. (A) Dilation of the ascending aorta (yellow arrow); (B) The intimal flap (yellow arrow). 


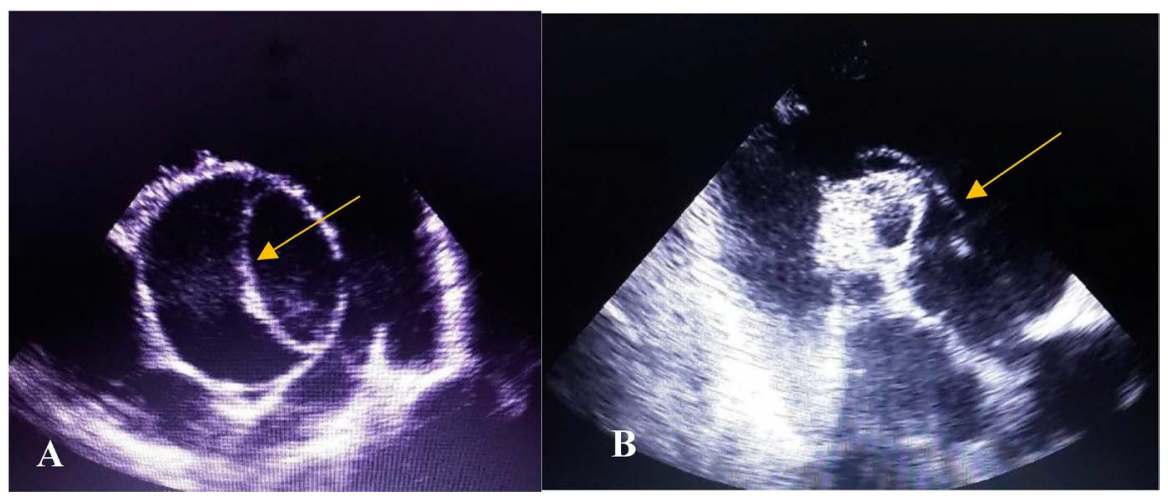

Figure 3. Transthoracic ultrasound. (A) Small parasternal axis a large vessel: the intimal flap (yellow arrow); (B) Sternal in the aorta: the intimal flap at the level of the descending aorta (yellow arrow).
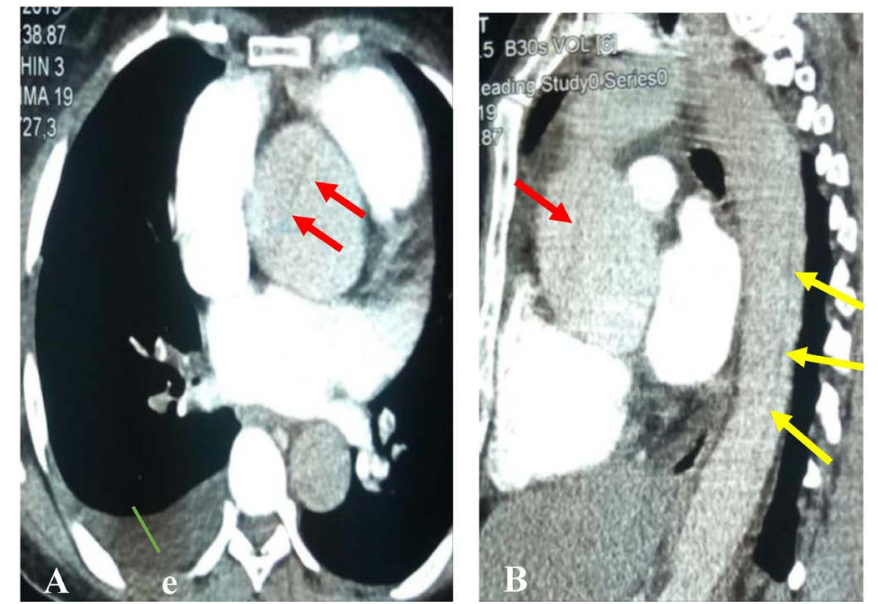

Figure 4. Thoracic CT scan showing dissection of the ascending aorta (red arrows) and the descending thoracic aorta (yellow arrows). (A) Axial section; (B) Sagittal reconstruction; e: right pleural effusion of low abundance (green arrow).
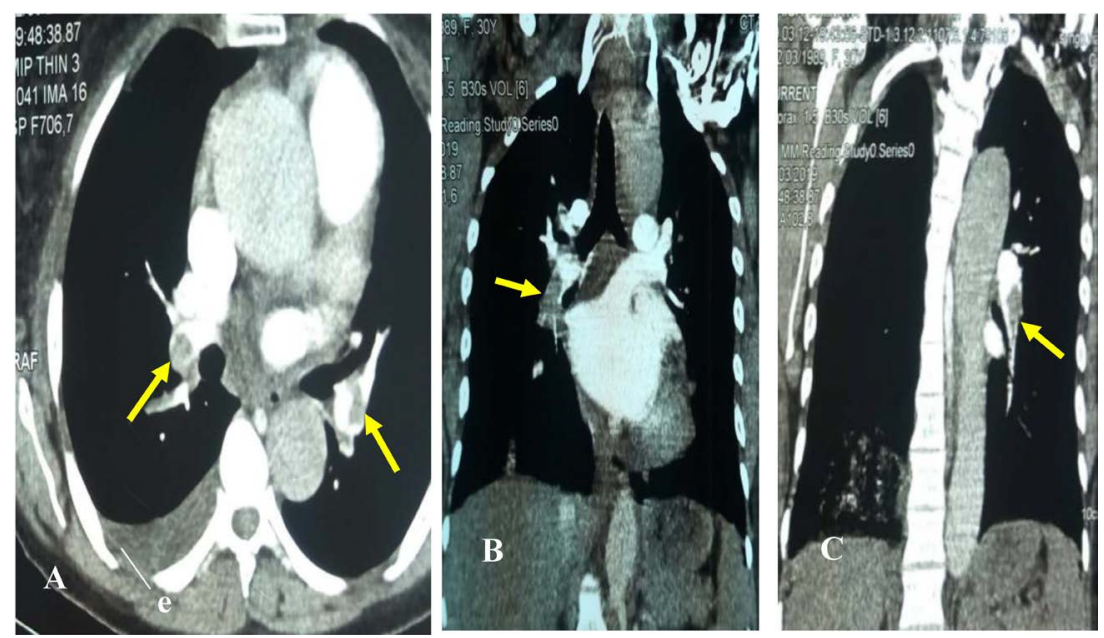

Figure 5. Thoracic CT scan showing thrombi in bilateral lobar arteries (yellow arrows). (A) Axial section; (B) and (C) Coronal reconstructions e: Pleural effusion (white arrow). (A) Coupe axiale; (B) and (C) Reconstructions coronales e: Epanchement pleural droit de faible abundance. 
The patient was put on oxygen therapy, Beta blocker (Bisoprolol $5 \mathrm{mg} 1$ tablet per day) for reduction of heart rate, diuretic (Lasilix $5 \mathrm{mg} 1$ comp per day) and IEC (Captopril $25 \mathrm{mg} 1$ comp per day) to reduce the signs of congestion, after confirmation of pulmonary embolism by CT angiography, by collegial decision it was put on anticoagulant (Lovenox at curative dose) for hemodynamic stabilization. In the absence of a surgical technical platform and the complexity of the case, preparations were underway for her evacuation to a specialized center for better care, unfortunately she died before the end of the evacuation process.

\section{Discussion}

Aortic dissection is a formidable medico-surgical emergency quickly putting life threatening into play. It is a complex and dynamic pathology with various pathophysiological mechanisms, likely to be the cause of a rupture or visceral ischemia. Aortic dissection is only suspected in half of the cases in the emergency room. Despite its low incidence, it can hide behind any chest or back pain. No element of the basic clinical presentation or paraclinical examination is sufficient to exclude it. On the other hend, brutal severe, migrating, heart breaking pain, a neurological or pulse deficit, or an enlargement of the mediastinum are specific data which initiate the continuation of the diagnostic process, with the performance of transesophageal ultrasound, CT scan or MRI (Magnetic resonance imaging) to confirm the diagnosis of an aortic dissection. The difficulty in diagnosing aortic dissection in the emergency room has been often reported in the literature. Emergency doctors suspect it only in half of the cases [7]. Especially since it concerns young patients or those with a nonthoracic clinical presentation in some cases [8]. Pulmonary embolism is defined by the occurrence of an acute or subacute partial or total occlusion of the trunk or one of the branches of the pulmonary artery by a foreign body most often circulating a fibrino-cruoric clot. There are thus variable clinical forms of pulmonary embolism depending on the severity, ranging from small thrombosis without clinical translation, to a massive, sudden and fatal form. Gradually, consecutive patient registers made it possible, among other things, to specify the risk factors for the disease and its most frequent source, deep vein thrombosis. Pulmonary embolism still remains a major challenge in medicine today despite progress in terms of prevention, diagnosis and treatment. It is often under estimated, under diagnosed and therefore under treated [1]. It is always to be considered as a frequent and serious disease [2], in particular in hemodynamically unstable patients [3], in the elderly [4], or in patients with severe underlying pathology in particular cardiopulmonary [5] [6], as in our case. In recent decades, specific diagnostic examinations, including lung scintigraphy and angiography, have helped define a category of patients less, severely affected, and with nonfatal pulmonary embolism. Despite this, the incidence has not decreased in recent years, the morbidity and mortality of pulmonary embolism are still high today. In fact, pulmonary embolism represents an estimated mortality between $6 \%$ and $17 \%$ in the quarter following 
the diagnosis, despite the use of anticoagulant therapy [9] [10] [11]. The pulmonary embolism associated with an acute aortic dissection constitutes a formidable cardiovascular emergency, with a grim prognosis as in our case. Few cases are reported in the literature. Abderrahmane Bakkali et al. reported a case to the cardiac surgery department of the CHU of Grenoble (France) in 2015, it is a 79-year-old patient, smoking, with a history of appendectomy in childhood, as well as an acute pericarditis treated in 1995. This patient presented in an abrupt way, an acute chest pain with type of constriction associated with a cyanosis of the extremities and a hemoptysis of average abundance progressing towards a syncope. The diagnosis of pulmonary embolism was suspected, anticoagulation treatment was started, after his admission additional examinations were carried out. The chest CT scan revealed a cracked ascending aortic aneurysm, hemorrhagic suffusion around the trunk of the pulmonary artery, right pulmonary artery, and bilateral pleural effusion with alveolar hemorrhage. The patient was transferred directly to the operating room for cardiac surgery according to the usual protocol, after a favorable post-operative course, he was released on the $16^{\text {th }}$ post-operative day [12]. A similar case was reported by GUINDO SA et al. in 2019 in Senegal, who was a 66-year-old patient with no health history, with the notion of long travel, received for prolonged chest pain for 3 days of sudden onset, maximum intensity right away, heart breaking, transfixing, migrating, the CT angiography showed a total thrombosis of the right pulmonary artery extending to part of the trunk and an ascending aorta dilated with an intimal flap extended from the butt to the abdominal aorta, surgery not being available and for lack of means for evacuation abroad, the development was lethal on the $2^{\text {nd }}$ day of hospitalization [13]. These patients presented the same diagnosis as our case, but they have their particularity, our patient was young female. The patient was on oral contraception (estrogen-progestin pills) after being replaced by the implant for a period of 10 years. The pills and the implant suppress ovulation. These contraceptive methods are at risk of side effects such as thromboembolism (phebitis or pulmonary embolism). The estrogens used in these preparations have effects on the coagulation system producing an increase in fibrinogen, coagulation factors (II, VII, VIII, IX, X, XII and XIII), a decrease in anticoagulant factors, antithrombin, protein $\mathrm{S}$ and acquired resistance to activated protein $\mathrm{C}$, inducing a prothrombotic state [14] [15]. Epidemiological data have shown that taking combined contraceptives increases the relative risk of thromboembolic disease by 2 to 6 times compared to women who do not take the combined pill [16] [17]. We believe that taking contraceptives for 10 years is an important risk factor in our case. One of the causes of aortic dissection is Marfan syndrome. It is a genetic disease that manifests as connective tissue damage, which is considered to be the supporting tissue for organs and fibers in the body. There is a genetic mutation in a protein called fibrillin, therefore the tissue containing this fibrillin is weaker, they do not support the body as much as they should. At the level of the aorta, it causes a loss of elasticity, subsequently dilation of the aorta 
with complications such as aortic insufficiency, aortic dissection or rupture of the aorta. The too loose skeleton and ligaments cause scoliosis and chest deformities. Myopia is frequent, displacement of the lens [18]. On physical examination of our case the extremities are thin, broad and deformed, the thorax deformed, the feet flat. The ophthalmological examination objectifies myopia. Cardiac ultrasound revealed an annulo-ectastic dilation of the aorta. These clinical and paraclinical signs made us think of Marfan syndrome, which will be the cause of aortic dissection. Given the predominance of signs of pulmonary embolism over aortic dissection, medical treatment has been established for hemodynamic stabilization: oxygen therapy, beta blocker (Bisoprolol $5 \mathrm{mg} 1$ tablet per day) for reduction of heart rate, diuretic (Lasilix $5 \mathrm{mg} 1$ comp per day) and the IEC (Captopril $25 \mathrm{mg} 1$ comp per day) to reduce the signs of congestion, after confirmation of the pulmonary embolism by the CT angiography, by collegial decision it was put on anticoagulant (Lovenox at curative dose). In the absence of a surgical technical plat form and the complexity of the case, preparations were underway for her evacuation to a specialized center for better care, unfortunately she died before the end of the evacuation process.

\section{Conclusion}

Aortic dissection is a pathology with a poor prognosis in the majority of cases; the diagnosis should be suspected in all patients with chest pain. We should always think about this pathology, when we suspect a pulmonary embolism. Despite the contribution of medical imaging to the diagnosis and management of aortic dissection and pulmonary embolism in recent years, we continue to receive patients in the complication phase, which makes it difficult to manage their pathologies.

\section{Conflicts of Interest}

The authors declare no conflicts of interest regarding the publication of this paper.

\section{References}

[1] Washington, L. and Gulsun, M. (2003) CT for Thromboembolic Disease. Current Problems in Diagnostic Radiology, 32, 105-126. https://doi.org/10.1016/S0363-0188(02)00006-3

[2] Silverstein, M.D., Heit, J.A., Mohr, D.N., Petterson, T.M., Fallon, W.M. and Melton, L.J. (1998) Trends in the Incidence of Deep Vein Thrombosis and Pulmonary Embolism: A 25-Year Population Based Study. Archives of Internal Medicine, 158, 585-593. https://doi.org/10.1001/archinte.158.6.585

[3] Kasper, W., Konstantinides, S., Geibel, A., Olschewski, M., Heinrich, F., Grosser, K.D., Rauber, K., Lversen, S., Redecker, M. and Kienast, L. (1997) Management Strategies and Determinants of Outcome in Acute Major Pulmonary Embolism: Results of a Multicenter Registry. Journal of the American College of Cardiology, 30, 1165-1171. https://doi.org/10.1016/S0735-1097(97)00319-7 
[4] Siddique, R.M., Siddique, M. and Rimm, A.A. (1998) Trends in Pulmonary Embolism Mortality in the US Elderly Population: 1984 through 1991. American Journal of Public Health, 88, 478-480. https://doi.org/10.2105/AJPH.88.3.478

[5] Harrington, K.J., Bateman, A.R., Syrigos, K.N., Rintoul, R., Bhidayasiri, R., McCormack, M. and Thomas, H. (1997) Cancer-Related Thromboembolic Disease in Patients with Solid Tumors: A Retrospective Analysis. Annals of Oncology, 8, 669-673. https://doi.org/10.1023/A:1008230706660

[6] Carson, J.L., Kelley, M.A., Duff, A., Weg, J.G., Fulkerson, W.J., Palevsky, H.L., Schwartz, J.S., Thompson, B.T., Popovich, J., Hobbins, T.E., et al. (1992) The Clinical Course of Pulmonary Embolism. The New England Journal of Medicine, 326, 1240-1245. https://doi.org/10.1056/NEJM199205073261902

[7] Spittell, P.C., Spittell, J.A., Joyce, J.W., Tajik, A.J., Edwards, W.D., et al. (2000) Clinical Features and Differential Diagnosis of Aortic Dissection: Experience with 236 Cases (1980 through 1990). Mayo Clinic Proceedings, 18, 46-51.

[8] Sullivan, P.R., Wolfson, A.B., Leckey, R.D. and Burke, J.L. (2000) Diagnosis of Acute Thoracic Aortic Dissection in the Emergency Department. The American Journal of Emergency Medicine, 18, 46-50. https://doi.org/10.1016/S0735-6757(00)90047-0

[9] La revue Prescrire (2000) Embolie pulmonaire: Quelques repères diagnostique. $R e$ vue Prescrire, 20, 476-477.

[10] Diehl, J.L. and Mercat, A. (1999) Gestion de l'embolie pulmonaire grave. Revue des Maladies Respiratoires, 16, 996-1006.

[11] Goldhaber, S. (1999) Treatment of Pulmonary Thromboembolism. Internal Medicine, 38, 620-625. https://doi.org/10.2169/internalmedicine.38.620

[12] Bakkali, A., Moncef, H., Mathieu, R., Aude, B. and Olivier, C. (2015) A Diagnostic Trap Simulating Pulmonary Embolism. Tunisie Medical, 93, 420.

[13] Guindo, S.A., et al. (2019) Double urgence associant une Dissection aortique aigue et Embolie pulmonaire d'évolution fatale; A propos d'un cas. Le journal Africain du thorax et des vaisseaux, 9, 99.

[14] WHO (1991) Task Force on Oral Contraceptives: A Multicenter Study of Coagulation and Haemostatic Variables during Oral Contraception: Variations with Four Formulations. British Journal of Obstetrics and Gynaecology, 98, 117-128. https://doi.org/10.1111/j.1471-0528.1991.tb15364.x

[15] Serfaty, D. (2007) Contraception, $3^{\text {ème }}$ Edition, Abrégés de médecine. Masson, Paris.

[16] Vanderbroucke, J.P., et al. (1994) Increased Risk of Venous Thrombosis in Oral-Contraceptive Users Who Are Carriers of Factor V Leiden Mutation. The Lancet, 344, 1453-1457. https://doi.org/10.1016/S0140-6736(94)90286-0

[17] WHO (1995) Venous Thromboembolic Disease and COC: Results of International Multicenter Case Control Study. WHO. Collaborative Study of Cardiovascular Disease and Steroid Hormone Contraception. The Lancet, 346, 1575-1582. https://doi.org/10.1016/S0140-6736(95)91926-0

[18] http://www.orpha.net/data/patho/Pub/fr/Marfan-FRfrPub109.pdf 